\title{
Applicability of the BET Method for Obtaining Surface Areas of Microporous Metal-Organic Frameworks
}

\author{
Krista S. Walton ${ }^{\dagger}$ and Randall Q. Snurr* \\ Department of Chemical and Biological Engineering, \\ Northwestern University, Evanston, IL 60208-3120 \\ *snurr@northwestern.edu \\ ${ }^{\dagger}$ Current Address: Department of Chemical Engineering, Kansas State University, \\ 1005 Durland Hall, Manhattan, KS 66506
}

\section{Supporting Information}

\section{Details of GCMC Simulations}

The MOFs and nitrogen molecules were represented in atomistic detail. Nitrogen-nitrogen interactions were modeled using the TraPPE potential. ${ }^{1}$ This model places a Lennard-Jones center on each nitrogen atom, a point charge of +0.964 on the center of mass of the molecule, and a charge of -0.482 on each nitrogen atom to simulate the molecule's quadrupole moment. The Lennard-Jones parameters for the framework atoms were taken from the DREIDING force field. ${ }^{2}$ Lorentz-Berthelot mixing rules were employed to calculate sorbate/framework parameters. Lennard-Jones interactions beyond $12.8 \AA \AA$ were neglected. All Lennard-Jones parameters are given in Table S1.

No charges were used for the MOF frameworks. Preliminary calculations for IRMOF-1 with charges included on the framework showed little effect of the framework charges on the adsorption isotherms. For the nitrogen-nitrogen Coulomb interactions, a cutoff based on the center-of-mass distance was used. This is justified (instead of the more expensive Ewald summation) because the $\mathrm{N}_{2}-\mathrm{N}_{2}$ quadrupole-quadrupole interaction is not long ranged.

Simulations were performed using the Music code. ${ }^{3}$ For each point on the isotherm, 10-35 million Monte Carlo steps were performed. The larger number of steps was required for equilibration at high adsorption amounts. Each step consisted of insertion of a new molecule, deletion of an existing molecule, or translation of an existing molecule. ${ }^{4}$ Typically, the first half of the run was used for equilibration, and the last half was used to calculate the ensemble averages.

The DREIDING forcefield was chosen for this work based upon its successful use in prior GCMC simulations of adsorption in MOFs. DREIDING has been shown to successfully reproduce adsorption isotherms for systems such as methane/IRMOFs ${ }^{5}$ and hydrogen/IRMOFs ${ }^{6}$. Also, the model works well in reproducing heats of adsorption for hydrogen in the IRMOF materials. ${ }^{6}$ In this work, we did not include detailed experimental isotherm comparisons 
because little to no nitrogen isotherm data are available for the MOFs we considered outside of the saturation region.

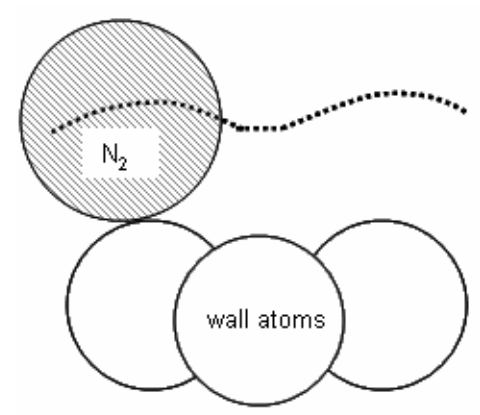

The accessible surface was calculated by performing many random insertions of a nitrogen-sized spherical probe molecule around each MOF atom. The insertions are done on a sphere whose radius is the sum of the radii of the probe and the framework atom. The accessible surface area is then obtained from the fraction of probe insertions that do not overlap with the other framework atoms. This gives the surface accessible to the center of a probe molecule as shown in Figure S1.

Figure S1. Definition of accessible surface area.

Table S1. Lennard-Jones Parameters used in GCMC Simulations

\begin{tabular}{l|cc} 
Atom Pair & $\sigma(\AA)$ & $\varepsilon / \mathrm{k}_{\mathrm{b}}(\mathrm{K})$ \\
\hline $\mathrm{N}-\mathrm{N}$ & 3.31 & 36.0 \\
$\mathrm{~N}-\mathrm{C}$ & 3.391 & 41.52 \\
$\mathrm{~N}-\mathrm{H}$ & 3.078 & 16.60 \\
$\mathrm{~N}-\mathrm{O}$ & 3.171 & 41.65 \\
$\mathrm{~N}-\mathrm{Zn}$ & 3.677 & 31.58
\end{tabular}




\section{$\underline{\text { Example BET Analysis }}{ }^{7-10}$ for IRMOF-1}

BET equation: $\quad v=\frac{c v_{m} x}{(1-x)[1+(c-1) x]}$

where $x=P / P_{0}, v$ is the volume of nitrogen adsorbed per gram of MOF at STP, $v_{m}$ is the monolayer capacity, and $c$ is related to the heat of adsorption.

The equation can be rewritten in the form:

$$
\frac{x}{v(1-x)}=\frac{1}{v_{m} c}+\frac{(c-1) x}{v_{m} c}
$$

A plot of the left-hand side of Eq (S2) vs. $x$ for IRMOF-1 is shown below for the isotherm given in Figure 2 of the main text. Notice that the line is fit to the low pressure isotherm data, which is well below the standard BET range of $0.05<x<0.3$.

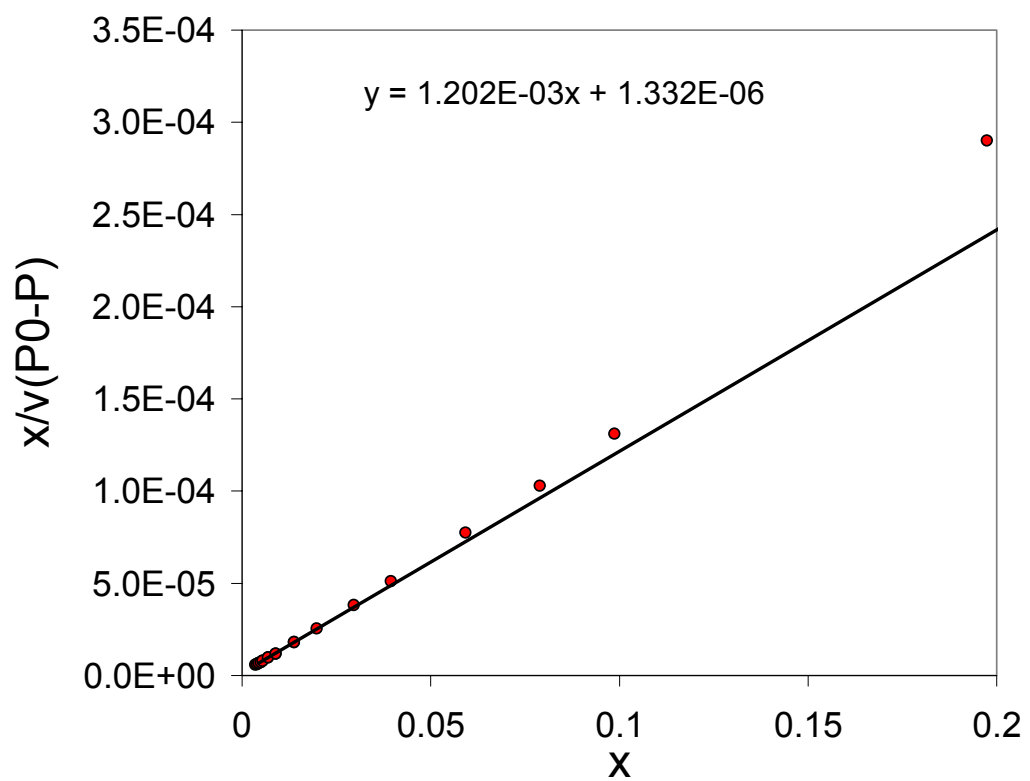

The slope and y-intercept of the line yield $c=903.4$ and $v_{m}=831.0 \mathrm{~cm}^{3}(\mathrm{STP}) / \mathrm{g}$.

The surface area is then calculated from:

$$
A=v_{m} \sigma_{0} N_{a v}
$$

where $\sigma_{0}$ is the cross-sectional area of nitrogen at liquid density (16.2 $\AA$ ) and $N_{a v}$ is Avogadro's number.

The resulting surface area is $3621 \mathrm{~m}^{2} / \mathrm{g}$. 


\section{References}

(1) Potoff, J.J.; Siepmann, J.I. AIChE J. 2001, 47, 1676.

(2) Mayo, S. L.; Olafson, B. D.; Goddard, W. A. J. Phys. Chem. 1990, 94, 8897.

(3) Gupta, A.; Chempath, S.; Sanborn, M.J.; Clark, L.A.; Snurr, R.Q. Molec. Simulation 2003, 29, 29.

(4) Frenkel, D.; Smit, B. Understanding Molecular Simulation: From Algorithms to Applications. $2^{\text {nd }}$ Ed., Academic Press: San Diego, 2002.

(5) Düren, T.; Sarkisov, L.; Yaghi, O.M.: Snurr, R.Q. Langmuir 2004, 20, 2683.

(6) Frost H., Düren T., Snurr, R.Q. J. Phys. Chem. B. 2006, 110, 9565.

(7) Brunauer, S.; Emmett, P. H.; Teller, E. J. Am. Chem. Soc. 1938, 60, 309.

(8) Rouquerol, J.; Llewellyn, P.; Rouquerol, F. Stud. Surf. Sci. Catal. 2007, 160, 49-56.

(9) Parra, J. B.; de Sousa, J. C.; Bansal, R. C.; Pis, J. J.; Pajares, J. A. Adsorpt. Sci. Technol. 1995, 12, 51 .

(10) Adamson, A. W.; Gast, A. P. Physical Chemistry of Surfaces, 6th ed., John Wiley \& Sons: New York, 1997. 